\title{
NOTES ON $\mathbf{P}_{\kappa} \lambda$ AND $[\lambda]^{\kappa}$
}

\author{
$\mathrm{By}$
}

\section{Yoshihiro ABE}

This paper consists of notes on some combinatorial properties. $\$ 1$ deals with $\lambda$-ineffability and the partition property of $P_{k} \lambda$ with $\lambda$ ineffable. In $\S 2$ we combine the flipping property and a filter investigated by Di Prisco and Marek to characterize huge cardinals.

We work in ZFC and the notations are standard. $P_{k} \lambda=\{x \subset \lambda:|x|<\kappa\}[\lambda]^{\kappa}=$ $\{x \subset \lambda:|x|=\kappa\}, D_{\kappa} \lambda=\left\{\{x, y\}: x, y \in P_{\kappa} \lambda\right.$ and $\left.x \subsetneq y\right\}$.

\section{$\S 1 P_{k} \lambda$ when $\lambda$ is ineffable.}

$\kappa$ is called $\lambda$-ineffable if for any function $f: P_{\kappa} \lambda \longrightarrow P_{\kappa} \lambda$ such that $f(x) \subset x$ for all $x \in P_{k} \lambda$, there is a subset $A$ of $\lambda$ such that the set $\left\{x \in P_{k} \lambda: A \cap x=f(x)\right\}$ is stationary. We abbreviate the following statement to $\operatorname{Part} *(\kappa, \lambda)$;

"For any function $F: D_{k} \lambda \longrightarrow 2$, there is a stationary homogeneous set $H$ i.e. $\left|F^{\prime \prime}\left([H]^{2} \cap D_{\kappa} \lambda\right)\right|=1 .^{\prime \prime}$

If $\operatorname{Part}^{*}(\kappa, \lambda)$, then $\kappa$ is $\lambda$-ineffable. We shall show the converse is true when $\lambda$ is ineffable.

Lemma 1. $X \subset P_{k} \lambda$ is closed unbounded iff $\left\{\alpha<\lambda: X \cap P_{k} \alpha\right.$ is closed unbounded in $\left.P_{\kappa} \alpha\right\}$ contains a closed unbounded subset of $\lambda$. Hence $S$ is stationary in $P_{\kappa} \lambda$ if $\left\{\alpha<\lambda: S \cap P_{\kappa} \alpha\right.$ is stationary in $\left.P_{\kappa} \alpha\right\}$ is a stationary subset of $\lambda$.

Theorem 2. Suppose that $\lambda$ is ineffable. If $\operatorname{Part}^{*}(\kappa, \alpha)$ for all $\alpha<\lambda$, then $\operatorname{Part*}(\kappa, \lambda)$.

Proof. Let $F: D_{\kappa} \lambda \longrightarrow 2$ and $F_{\alpha}=F \uparrow D_{\kappa} \alpha$ for every $\alpha<\lambda$. By our assumptions, there is a stationary subset $A_{\alpha}$ of $P_{\kappa} \alpha$ such that

$$
F^{\prime \prime}\left(\left[A_{\alpha}\right]^{2} \cap D_{\kappa} \alpha\right)=\left\{k_{\alpha}\right\}, k_{\alpha} \in\{0,1\} .
$$

Since $\lambda$ is ineffable, we can find an $A \subset P_{k} \lambda$ so that

$$
S=\left\{\alpha<\lambda: A_{\kappa}=A \cap P_{\kappa} \alpha\right\} \text { is stationary in } \lambda .
$$

Received September 30, 1985. 
A is stationary by Lemma 1 .

Let $t, u \in[A]^{2} \cap D_{x} \lambda$. Since $S$ is unbounded in $\lambda$, there is a member of $S, \alpha$ such that both $t$ and $u$ are in $\left[A_{\alpha}\right]^{2} \cap D_{\kappa} \alpha$. Hence $F(t)=F(u)=k_{\alpha}$. So, A is a stationary homogeneous set for $F$.

Definition. $\kappa$ is $\lambda$-almost ineffable if for any function $f: P_{x} \lambda \longrightarrow P_{\alpha} \lambda$ such that $f(x) \subset x$ for all $x \in P_{x} \lambda$, there is a subset $A$ of $\lambda$ such that the set $\left\{x \in P_{k} \lambda\right.$ : $A \cap x=f(x)\}$ is unbounded.

Theorem 3. Suppose that $\lambda$ is almost ineffable. Then $\kappa$ is $\lambda$-almost ineffable iff $\kappa$ is $\alpha$-almost ineffable for all $\alpha<\lambda$.

ProOF. $\longrightarrow$ is proved by the same argument as the lemma in Magidor [9] p.p. 281.

$(\longleftarrow)$ Let $f: P_{\star} \lambda \longrightarrow P_{\varsigma} \lambda$ and $f(x) \subset x$ for all $x \in P_{\star} \lambda$. Considering a function $f \nmid P_{\star} \alpha$ and using $\alpha$-ineffability, we get an $A_{\alpha} \subset \alpha$ for every $\alpha<\lambda$ such that

$$
X_{\alpha}=\left\{x \in P_{\alpha} \alpha: f(x)=x \cap A_{\alpha}\right\} \text { is unbounded in } P_{\alpha} \alpha \text {. }
$$

Using now the almost ineffability of $\lambda$, there is an $A \subset \lambda$ so that

$$
S=\left\{\alpha<\lambda: A_{\alpha}=A \cap \alpha\right\} \text { is unbounded in } \lambda .
$$

Let $X=\left\{x \in P_{\alpha} \lambda: f(x)=x \cap A\right\}$. If $\alpha \in S$ and $x \in P_{\alpha} \alpha$, then $x \cap A_{\alpha}=x \cap A \cap \alpha=x \cap A$. Hence $X_{\alpha} \subset X \cap P_{\kappa} \alpha$ for every $\alpha \in S$. This gives

$$
\left\{\alpha<\lambda: X \cap P_{\star} \alpha \text { is unbounded in } P_{\star} \alpha\right\} \text { is unbounded in } \lambda \text {. }
$$

Thus $X$ is unbounded in $P_{k} \lambda$.

Corollary 4. The following are equivalent for $\kappa<\lambda$ with $\lambda$ ineffable.

(a) $\operatorname{Part*}(\kappa, \alpha)$ for all $\alpha<\lambda$.

(b) $\operatorname{Part}^{*}(\kappa, \lambda)$.

(c) $\kappa$ is $\lambda$-ineffable.

(d) $\kappa$ is $\alpha$-ineffable for all $\alpha<\lambda$.

(e) $\kappa$ is $\alpha$-almost ineffable for all $\alpha<\lambda$.

(f) $\kappa$ is $\lambda$-almost ineffable.

(g) $\kappa$ is $\alpha$-supercompact for all $\alpha<\lambda$.

Proof. (a) $\longrightarrow($ b) is Theorem 1. (b) $\longrightarrow($ c) is Theorem 2 in Magidor [9]. $(c) \longrightarrow(d)$ is the lemma also in [9]. (d) $\longrightarrow$ (e) is trivial. (e) $\longleftrightarrow(f)$ is Theorem 3. (e) $\longrightarrow(\mathrm{g})$ is by Carr's result: If $\kappa$ is $2^{\alpha<\kappa}$-shelah, then $\kappa$ is $\alpha$-supercompact. ( $\kappa$ is $\alpha$-shelah if $\kappa$ is $\alpha$-almost ineffable.) See [3]. 
On the coding of $P_{\kappa} \lambda$, there are works of $Z$ wicker [14] and Shelah [12]. The author can not answer this question.

Question 5 . Is there a function $t: \lambda \longrightarrow P_{\kappa} \lambda$ such that for any stationary subset $A$ of $\lambda, t^{\prime \prime} A$ is stationary in $P_{a} \lambda$.

It is, of course, true if $\kappa=\lambda$. In fact let $t=i d . \mid \kappa$. The question is interesting when $\lambda$ is ineffable.

Proposition 6. If $\lambda$ is ineffable and there is a $t: \lambda \longrightarrow P_{x} \lambda$ such that $t^{\prime \prime} A$ is stationary for any stationary subset $A$ of $\lambda$, then $\kappa$ is $\lambda$-ineffable.

Proof. Suppose that $f: P_{\kappa} \lambda \longrightarrow P_{\kappa} \lambda$ and $f(x) \subset x$ for all $x \in P_{\kappa} \lambda$. Let $A_{\alpha}=\{\beta<\alpha$ : $\beta \in f(t(\alpha))\}$. Since $\lambda$ is ineffable, there is a stationary subset $S$ of $\lambda$ and $A \subset \lambda$ so that $A_{\alpha}=A \cap \alpha$ for all $\alpha \in S$.

$B=t^{\prime \prime} S$ is stationary and for any $x \in B$ there is an $\alpha_{x} \in S$ such that $x=t\left(\alpha_{x}\right)$. Hence $f(x) \cap \alpha_{x}=A \cap \alpha_{x}$.

Let $B^{\prime}=\{x \in B: f(x) \neq A \cap x\}$ and $\delta_{x}=$ the least ordinal in $f(x) \Delta(A \cap x) . \quad \delta_{x} \in x$ for all $x \in B^{\prime}$.

Soppose that $B^{\prime}$ is stationary. There is an ordinal $\delta<\lambda$ such that $C=\left\{x \in B^{\prime}\right.$ : $\left.\delta_{x}=\delta\right\}$ is stationary.

$$
\forall x \in C(f(x) \cap(\delta+1) \neq A \cap(\delta+1)) .
$$

So,

$$
\begin{aligned}
& \forall x \in C\left(\alpha_{x}<\delta\right) . \\
& \left|\left\{\alpha_{x}: x \in C\right\}\right| \geq|C|=\lambda^{<x} \geqslant \lambda .
\end{aligned}
$$

Thus there is an $x \in C$ such that $\delta<\alpha_{x}$.

Hence $\{x \in B: f(x)=A \cap x\}$ is stationary.

REMARK. $t^{\prime \prime} A$ is a stationary subset which splits into $\lambda$ disjoint stationary subsets. Gitik constructed a model of $Z F C$ in which there is a stationary set that can not be splitted into $\lambda$ disjoint stationary subsets in [6].

\section{$\S 2[\lambda]^{\kappa}$ when $\boldsymbol{x}$ is huge.}

Let $j: V \longrightarrow M$ be a huge embedding with critical point $\kappa$ and $j(\kappa)=\lambda$ in this section.

At first we recall a filter on $[\lambda]^{\kappa}$ investigated by Di Prisco and Marek in [5]. It is analogous to the closed unbounded filter on $P_{x} \lambda$. 
Definition. For $X \subset P_{\kappa} \lambda$, define $A_{X}$, the basic set generated by $X$, as follows: $A_{X}=\left\{x \in[\lambda]^{\kappa}: x\right.$ is the union of an increasing $\kappa$-chain of elements of $\left.X\right\}$. Define $F_{\kappa, \lambda}$ by

$A \in F_{\kappa, \lambda}$ iff there is a closed unbounded $X \subset P_{\kappa} \lambda$ such that $A_{X} \subset A$.

Theorem (Di Prisco, Marek, Baumgartner)

$F_{\kappa, \lambda}$ is the least $\kappa$-complete, normal, fine filter on $[\lambda]^{\kappa}$. If $U$ is the normal ultrafilter on $[\lambda]^{x}$ induced by $j$, then every set in $F_{x, \lambda}$ is in $U$. In this case $F_{x, \lambda}$ is not $\kappa^{+}$-complete.

$X \subset[\lambda]^{\kappa}$ is unbounded if $\forall x \in[\lambda]^{\kappa} \exists y \in X(x \subset y) . \quad X$ is $F_{\kappa, \lambda}$ stationary if $X \cap Y \neq 0$ for all $Y \in F_{\kappa, \lambda}$.

Proposition 1. Any $X \in F_{x, \lambda}$ is unbounded.

Proof. There is a $C \subset P_{x} \lambda$ that is closed unbounded and $C_{X} \subset X$. Let $a \in[\lambda]^{k}$ and $f: \kappa \longrightarrow$ a be a bijection, $x_{\alpha}=f^{\prime \prime} \alpha$ for all $\alpha<\kappa$. We can find, using induction, $y_{\alpha} \in C$ such that $y_{\alpha} \nsupseteq x_{\alpha} \cup\left\{y_{\beta}: \beta<\alpha\right\}$ for every $\alpha<\kappa$.

$\left\{y_{\alpha} \mid \alpha<\kappa\right\} \subset C$ is a $\kappa$-chain and $x=\bigcup\left\{x_{\alpha}: \alpha<\kappa\right\} \subset \bigcup\left\{y_{\alpha}: \alpha<\kappa\right\}=y \in C_{X} \subset X$.

Next proposition shows the situation is different from $P_{x} \lambda$.

Proposition 2. If $\kappa$ is huge, there is a $F_{\kappa, \lambda}$-stationary set that is not unbounded.

Proof. $(\lambda)^{x}=\left\{x \in[\lambda]^{\kappa}\right.$ : the order type of $x$ is $\left.\kappa\right\}$ is in $U$. Clearly $(\lambda)^{x}$ is not unbounded.

Moreover, we shall show that there is a $F_{a, \lambda}$-stationary set $S$ such that for any $x, y$ in $S, x \nsubseteq y$. Thus, partition property may not be directry extended to $[\lambda]^{x}$ as for $P_{x} \lambda$.

Definition. $f$ is a $\omega$-Jonsson function over a set $x$ iff $f:{ }^{\omega} x \longrightarrow x$ and whenever $y \subset x$ and $|y|=|x|, f^{\prime \prime \omega} y=x$.

Lemma 3. Let $U$ be the normal ultrafilter on $[\lambda]^{\star}$ induced by $j$ and $f$ is a $\omega$-Jonsson function over $\lambda$. Then $\left\{x \in[\lambda]^{\kappa}: f \uparrow^{\omega} x\right.$ is $\omega$-Jonsson over $\left.x\right\} \in U$.

Proof. The same argument as a normal ultrafilter on $P_{\star} \lambda$ can be carried out. Let $e: V \longrightarrow N \simeq V^{[]^{k}} / U$ and $X \subset e^{\prime \prime} \lambda$ with $|X|=\left|e^{\prime \prime} \lambda\right|=\lambda$. Since $Y=e^{-1}(X) \subset \lambda$ and $|Y|=\lambda, f^{\prime \prime \omega} Y=\lambda$. So,

$$
\forall \alpha<\lambda \exists s \epsilon^{\omega} Y(\alpha=f(s))
$$


This implies

$$
\forall \alpha \in e^{\prime \prime} \lambda \exists s \epsilon^{\omega} Y(\alpha=e(f)(e(s)))
$$

Since $e(s)=e^{\prime \prime} s \epsilon^{\omega} X$,

$$
e(f)^{\prime \prime \omega} X=e^{\prime \prime \lambda}
$$

Hence $e(f) \Gamma^{\omega} e^{\prime \prime} \lambda$ is $\omega$-Jonsson over $e^{\prime \prime} \lambda$.

Thus $\left\{x: f \uparrow^{\omega} x\right.$ is $\omega$-Jonsson over $\left.x\right\} \in U$.

Theorem 4. There is an $A \in U$ such that for every pair $x, y$ in $A, x \nsubseteq y$.

Proof. Let $f$ be a $\omega$-Jonsson function over $\lambda$ and $A=\left\{x \in[\lambda]^{\pi}: f \uparrow^{\omega} x\right.$ is $\omega$ Jonsson over $x\} \in U$.

Suppose $y \nsubseteq x \in A$. Since $|x|=|y|, f^{\prime \prime \omega} y=x$. But $f^{\prime \prime \omega} y \subset y$.

\section{§ 3 Flipping properties and huge cardinals, partition properties of $\boldsymbol{P}_{k} \lambda$.}

Flipping properties wrer first studied by Abramson, Harrington, Kleinberg and Zwicker in [1] and turned out to be another form of large cardinal property. Di Prisco and $Z$ wicker [4] extended this line to supercompactness. More precisely, they gave a new type of flipping properties equivalent to $\lambda$-ineffability and $\lambda$-mildly ineffability. We shall introduce an analogous type properties and discuss the relationship with huge cardinals.

Definition. If $t: \lambda \longrightarrow P\left([\lambda]^{\kappa}\right)$, we call $t^{\prime}$ a flip of $t\left(t^{\prime} \sim t\right)$ if $t^{\prime}: \lambda \longrightarrow P\left([\lambda]^{\kappa}\right)$ and for all $\alpha<\lambda, t^{\prime}(\alpha)=t(\alpha)$ or $t^{\prime}(\alpha)=[\lambda]^{\kappa}-t(\alpha) . \quad \operatorname{Flip}(\kappa, \lambda) \equiv \forall t: \lambda \longrightarrow P\left([\lambda]^{\alpha}\right) \exists t^{\prime} \sim t$ such that $\Delta t_{\alpha<\lambda}^{\prime}(\alpha)$ is $F_{\kappa, \lambda}$-stationary. Inef $(\kappa, \lambda) \equiv$ for any function $f:[\lambda]^{\kappa} \longrightarrow[\lambda]^{\kappa}$ such that $f(x) \subset x$ for all $x \in[\lambda]^{\kappa}$, there is a subset $A$ of $\lambda$ such that the set

$$
\left\{x \in[\lambda]^{\kappa}: A \cap x=f(x)\right\} \text { is } F_{\kappa, \lambda} \text {-stationary. }
$$

THEOREM 1. (i) $\operatorname{Flip}(\kappa, \lambda)$ iff $\operatorname{Inef}(\kappa, \lambda)$.

(ii) If $\operatorname{Flip}\left(\kappa, 2^{\alpha^{\kappa}}\right)$, then there is a huge embedding $j$ such that $\kappa$ is the critical point and $j(\kappa)=\lambda$.

(iii) If $j: V \longrightarrow M$ is a huge embedding with the critical point $\kappa$ such that $j(\kappa)=\lambda$, then $\operatorname{Flip}(\kappa, \lambda)$.

Proof (i) Assume that $\operatorname{Flip}(\kappa, \lambda)$ and $f:[\lambda]^{\kappa} \longrightarrow[\lambda]^{\kappa}$ such that $f(x) \subset x$ for all $x \in[\lambda]^{\kappa}$. Define $t: \lambda \longrightarrow P\left([\lambda]^{\kappa}\right)$ by

$$
t(\alpha)=\left\{x \in[\lambda]^{\kappa}: \alpha \in f(x)\right\} .
$$




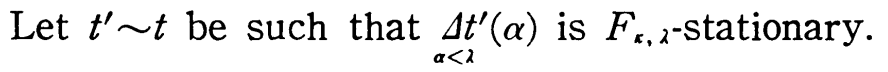

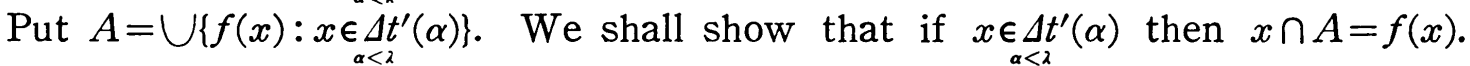
Obviously $f(x) \subset x \cap A$. If $\alpha \in x \cap A$, then there is a $y \in \Delta<t^{\prime}(\beta)$ so that $\alpha \in f(y)$. Since $\alpha \in f(y), y \in t(\alpha)$ and $\alpha \in y$. Hence $t^{\prime}(\alpha)=t(\alpha)$. Now $\alpha \in x \in \underset{\beta<\lambda}{\Delta t^{\prime}(\beta)}$ and $t^{\prime}(\alpha)=t(\alpha)$. This gives $x \in t(\alpha)$. Hence $\alpha \in f(x)$.

Conversely, let $t: \lambda \longrightarrow P\left([\lambda]^{x}\right)$. Define $f:[\lambda]^{\kappa} \longrightarrow[\lambda]^{x}$ by

$$
f(x)=\{\alpha \in x: x \in t(\alpha)\} .
$$

There is a subset $A$ of $\lambda$ such that $B=\left\{x \in[\lambda]^{\kappa}: x \cap A=f(x)\right\}$ is $F_{\kappa, \lambda}$-stationary. Define $t^{\prime}: \lambda \longrightarrow P\left([\lambda]^{\alpha}\right)$ by $t^{\prime}(\alpha)=t(\alpha)$ if $\alpha \in A$ and $t^{\prime}(\alpha)=[\lambda]^{\alpha}-t(\alpha)$ if $\alpha \notin A$.

Suppose $x \in S$ and $\alpha \in x$. If $\alpha \in A$, then $\alpha \in f(x)$ hence $x \in t(\alpha)=t^{\prime}(\alpha)$. If $\alpha \notin A$, then $\alpha \notin f(x)$ hence $x \notin t(\alpha)$. So $x \in t^{\prime}(\alpha)$. Now we have shown $S \subset \Delta t_{\alpha<\lambda}^{\prime}(\alpha)$, which must be $F_{x, 2}$-stationary.

(ii) Let $\gamma=2^{\alpha^{\kappa}}$ and $\left\{A_{\alpha}: \alpha<\gamma\right\}$ be an enumeration of $P\left([\lambda]^{\kappa}\right)$. Define $t: \gamma \longrightarrow$

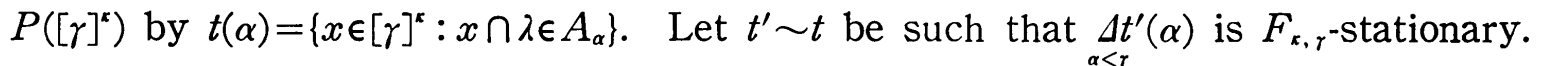

A filter $U$ on $[\lambda]^{\alpha}$ is defined by $A_{\alpha} \in U$ iff $t^{\prime}(\alpha)=t(\alpha)$. We shall show in fact $U$ is a normal ultrafilter. The fact that for any $a \in P_{x} \gamma$ the set $\left\{x \in[\gamma]^{\star}: a \subset x\right\}$ is a member of $F_{\kappa, r}$ is often used.

(1) $A_{\alpha} \in U \wedge A_{\alpha} \subset A_{\beta} \longrightarrow A_{\beta} \in U$.

There is a $x \in \Delta t^{\prime}(\xi)$ such that $\{\alpha, \beta\} \subset x$. Since $x \in t^{\prime}(\alpha)=t(\alpha), x \cap \lambda \in A_{\alpha} \subset A_{\beta}$. Thus $x \in t(\beta)$. Hence $t^{\prime}(\beta)=t(\beta)$.

(2) $U$ is $\kappa$-complete.

Suppose $\left\{B_{\alpha}: \alpha<\delta\right\} \subset U(\delta<\kappa)$ and $f: \delta \longrightarrow \gamma$ such that $B_{\alpha}=A_{f(\alpha)}$ for all $\alpha<\delta$. Let $A_{\eta}=\bigcap_{\alpha<\delta} B_{\alpha}$.

There is a $x \in \Delta t^{\prime}(\xi)$ such that $\{\eta\} \cup f^{\prime \prime} \delta \subset x$. For all $\alpha<\delta, x \in t^{\prime}(f(\alpha))=t(f(\alpha))$, so $x \in \bigcap_{\alpha<\delta} A_{f(\alpha)}$. Hence $x \cap \lambda \in A_{\eta}$. This shows $x \in t(\eta)$ and $t^{\prime}(\eta)=t(\eta)$.

(3) For any $\alpha<\lambda,\left\{x \in[\lambda]^{\alpha}: \alpha \in x\right\} \in U$.

Let $A_{\beta}=\left\{x \in[\lambda]^{x}: \alpha \in x\right\}$. $t(\beta)=\left\{x \in[\gamma]^{\alpha}: \alpha \in x \cap \lambda\right\}=\left\{x \in[\gamma]^{x}: \alpha \in x\right\} \in F_{k, \gamma}$. There is a

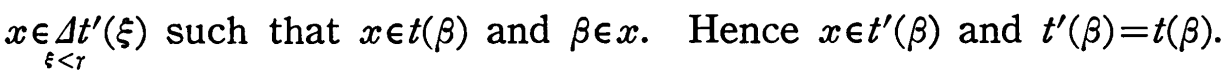

(4) $U$ is an ultrafilter.

Obviously $\phi \notin U$. So we have to show only that if $A \notin U$, then $[\lambda]^{\kappa}-A \in U$. Suppose that $A_{\alpha} \notin U . \quad t^{\prime}(\alpha)=[\gamma]^{\kappa}-t(\alpha)$. Let $[\lambda]^{\kappa}-A_{\alpha}=A_{\beta}$. There is a $x \in \Delta t_{\xi<\gamma}^{\prime}(\xi)$ such that $\{\alpha, \beta\} \subset x$. Since $x \in t^{\prime}(\alpha)=[\gamma]^{\alpha}-t(\alpha), x \cap \lambda \notin A_{\alpha}$. Hence $x \cap \lambda \in A_{\beta}$ and $x \in t(\beta)$. Thus $t^{\prime}(\beta)=t(\beta)$.

(5) $U$ is normal.

Suppose that $\left\{B_{\alpha}: \alpha<\lambda\right\} \subset U$. Let $f: \lambda \longrightarrow \gamma$ be such that $B_{\alpha}=A_{f(\alpha)}$ for all $\alpha<\lambda$, 
and $\underset{\alpha<2}{\Delta B_{\alpha}}=A_{\beta}$.

Note that $X=\left\{x \in P_{\mathrm{r} \gamma}: \forall \alpha \in x \cap \lambda(f(\alpha) \in x)\right\}$ is a closed unbounded subset of $P_{\boldsymbol{r}} \gamma$. Let $C=A_{X}=\left\{y \in[\gamma]^{\alpha}: \exists D \subset X(D\right.$ is a $\kappa$-chain, $\left.y=\bigcup D)\right\}$. Then $C \in F_{s, r}$.

If $y \in C$ and $\alpha \in y \cap \lambda$, there is an $x \in D$ such that $\alpha \in x \cap \lambda$ and $x \subset y$. Hence $f(\alpha) \in x \subset y$. Now we have got that for any $y \in C$, if $\alpha \in y \cap \lambda$ then $f(\alpha) \in y$.

There is a $y \in \Delta t^{\prime}(\xi)$ such that $y \in C$ and $\beta \in y$. For all $\alpha \in y \cap \lambda, f(\alpha) \in y$ and $y \in t^{\prime}(f(\alpha))=t(f(\alpha))$, hence $y \cap \lambda \in A_{f(\alpha)}$.

If $t^{\prime}(\beta)=[\gamma]^{x}-t(\beta), y \cap \lambda \notin A_{\beta}=\Delta B_{\alpha}$. So, there is an $\alpha \in y \cap \lambda$ such that $y \cap \lambda \notin B_{\alpha}=$ $A_{f(\alpha)}$. Contradiction. Hence $t^{\prime}(\beta)=t(\beta)$.

(iii) Let $U$ be the normal ultrafilter on $[\lambda]^{\kappa}$ induced by $j, t: \lambda \longrightarrow P\left([\lambda]^{\pi}\right)$. Define $t^{\prime}: \lambda \longrightarrow P\left([\lambda]^{\alpha}\right)$ as follows. $t^{\prime}(\alpha)=t(\alpha)$ if $t(\alpha) \in U$, and $t^{\prime}(\alpha)=[\lambda]^{\alpha}-t(\alpha)$ if $t(\alpha) \notin U$.

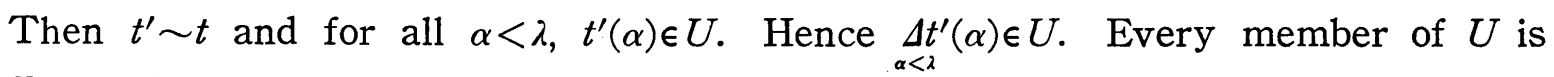
$F_{r, \lambda}$-stationary.

Next the author tried to express the partition property of $P_{x} \lambda$ in the form of a flipping propertie. (Though it does not seem successful.)

PRopposition 2. The followings are equivalent.

(a) $\operatorname{Part}^{*}(\kappa, \lambda)$.

(b) For any $t: P_{s} \lambda \longrightarrow P\left(P_{k} \lambda\right)$, there are $t^{\prime} \sim t$ and a stationary set $X$ such that if $\{x, y\} \in D_{\kappa} \lambda \cap[X]^{2}$ then $y \in t^{\prime}(x)$.

Proof. $(a) \longrightarrow(b)$. Define $F: D_{\kappa} \lambda \longrightarrow 2$ by $F(x, y)=0$ if $y \in t(x)$ and $F(x, y)=1$ otherwise. Let $X$ be a stationary homogeneous set for $F$. When $F^{\prime \prime}\left([X]^{2} \cap D_{k} \lambda\right)=$ $\{0\}, t^{\prime}=t$. If $F^{\prime \prime}\left([X]^{2} \cap D_{\mathrm{k}} \lambda\right)=\{1\}$, let $t^{\prime}(x)=P_{\mathrm{\kappa}} \lambda-t(x)$ for all $x \in X$.

(b) $\longrightarrow$ (a). Put $t(x)=\{y: F(x, y)=0\}$. There are $t^{\prime} \sim t$ and a stationary set $X$ such that if $x \nsubseteq y \in X$ then $x \in t^{\prime}(y)$.

Let $X_{1}=\left\{x \in X: t^{\prime}(x)=t(x)\right\}$ and $X_{2}=\left\{x \in X: t^{\prime}(x)=P_{x} \lambda-t(x)\right\}$. Either $X_{1}$ or $X_{2}$ is stationary and both of them are homogeneous set for $F$.

We add easy obervations at the end of this paper.

Definition. A stationary coding set for $P_{\mathrm{r}} \lambda$ (an "SC") consists of a stationary set $A \subset P_{\kappa} \lambda$ together with a $1: 1$ function $c: A \longrightarrow \lambda$ (called the coding function) satisfying that for each $x, y \in A$

$$
x \nsubseteq y \leftrightarrow c(x) \in y .
$$

Proposition 3. If $\operatorname{Part}^{*}(\kappa, \lambda)$, then an $S C$ exists. (This is also seen in $Z$ wicker [14]. The author considered this property without a word an "SC".)

Proof. Let $F(x, y)=0$ if $c(x) \in y$ and $F(x, y)=1$ otherwise, for any $1: 1$ func- 
tion $c: P_{\kappa} \lambda \longrightarrow \lambda$.

Definition. $X \subset P_{x} \lambda$ is prestationary iff for any choice function on $X$ is constant on some unbounded set $S \subset X$.

This definition makes sense. In fact,

Lemma 4. (Menas in [10]) There is a prestationary set that is not stationary.

LemmA 5. If $X$ is prestationary, then $\{x \in X: a \subset x\}$ is also prestationary for all $a \in P_{x} \lambda$.

Definition. $\quad \operatorname{Part}^{*}(\kappa, \lambda)$ iff any partition of $P_{\kappa} \lambda$ has a prestationary homogeous set.

Theorem 6. If $\operatorname{wPart}^{*}(\kappa, \lambda)$, then $\kappa$ is almost $\lambda$-ineffable.

Proop. Magidor's proof of Theorem 2 in [9] can be carried out. What we really need is a homogeneous set $H$ such that for any choice function $f$ there is an unbounded subset $T$ of $H$ so that

$$
\forall x \in T \exists y \in T(x \nsubseteq y \text { and } f(x) \geqslant f(y)) .
$$

\section{References}

[1] Abramson, F. G. Harrington, L. A. Kleinberg, E. M. and Zwicker, W. S. Flipping properties: a unifying thread in the theory of large cardinals, Ann. Math. Logic 12 (1977), 37-58.

[2] Carr, D. M. The minimal normal filter on $P_{x} \lambda$, Proc. Amer. Math. Soc. 86 (1982), 216320.

[ 3 ] Carr, D. M., The structure of ineffability properties of $P_{k} \lambda, \operatorname{tr}$ appear.

[4] Di Prisco C., and Zwicker, W., Flipping properties and supercompact cardinals, Fund. Math. 69 (1980), 31-36.

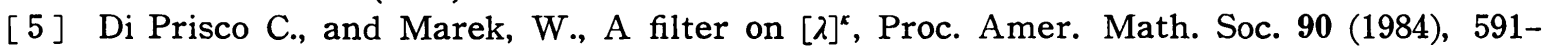
598.

[6] Gitik, M., Nonsplitting subset of $\mathrm{P}_{\boldsymbol{x}}\left(\pi^{+}\right)$, J. Sym. Logic 50 (1985), 881-894.

[7] Jech, T. J., Some combinatorial problems concerning uncountable cardinals, Ann. Math. Logic 7 (1973), 165-198.

[8] Kanamori A., and Magidor M., The development of large cardinals axioms in set theory, Higer set theory, Lecture Notes in Mathematics, 699, Springer-Verlag, Berlin and New York, 99-276.

[9] Magidor M., Combinatorial characterization of supercompact cardinals, Proc. Amer. Math. Soc. 42 (1974), 327-359.

[10] Menas, T. K., On strong compactness and supercompactness, Ann. Math. Logic 7 (1974), 327-359.

[11] Menas T. K., A combinatorihl property of $P_{\kappa} \lambda$, J. Sym. Logic 41 (1976), 225-234. 
[12] Shelah S., The existence of coding sets, to appear.

[13] Solovay R. M., Reinhaldt W. N., and Kanamori A., Strong axioms of infinity and elementary embeddings, Ann. Math. Logic 13 (1978), 379-419.

[14] Zwicker W., $\mathrm{P}_{\kappa} \lambda$ combinatorics $\mathrm{I}$ : stationary coding sets rationalize the club filter, Contemporary Mathematics 31 (1984), 243-259.

Fukushima Technical College

Taira, Kamiarakawa, Nagao 30

Iwaki, 970 